\title{
Integración y Regionalización de la Información
}

Kira Tarapanoff ${ }^{\star}$

\begin{abstract}
RESUMEN
Considera las posibilidades de regionalización de la información para América Latina, en especial para los paises que integran el MERCOSUR. Analiza las propuestas del COMUTEX (sistema de accesos al documento primario para América Latina) y de su instrumento de apoyo, el CAPSALC (Catálogo Colectivo para América Latina y el Caribe) cómo acciones concretas para compartir recursos y regionalización de la información, que podrán ser viabilizadas con la participación comunitaria. Concluye con las necesidades de padronización bibliográfica y de formatos legibles por máquina, lenguaje de máquina común, legislación específica, política de cooperación unificada y centralizada, así como política, valor y tasa de conversión de cupones unificados.
\end{abstract}

\section{ABSTRACT}

The author discusses the posibilities of regionalization of information for Latin America, specially of Countries which form MERCOSUR. Proposals of COMUTEX (Access System to the Primary Document for Latin America), and the CAPSALC (Collective Catalogue for Latin America and the Caribbean), a backup device of the former, are analyzed as concrete actions to share resources and information regionalization. They could be feasible through community participation, She concludes with the need of bibliographic listings and machine readable formats, common machine language, specific regulations, unified and centralized cooperation policy, as well as unified coupon's policy, value and exchange rate.

\section{EL CONTEXTO SOCIAL E INFORMACIÓN DEL DESARROLLO}

$\boldsymbol{B}$ ibliotecas y otros tipos de unidades de información son agentes sociales de desarrollo. Poseen gran efecto multiplicador a nivel local, nacional e internacional; sus productos y servicios son factores de desarrollo, pudiendo, por ejemplo, traer como valor agregado $^{1}$ las ventajas competitivas para quien retiene la información "de más". El beneficio social y económico que adviene esas unidades de información es inmensurable. Congregan en un solo local o punto de referencia documentos y datos que pueden ser utiliza- dos, independientemente del tiempo o del espacio, por un número siempre renovado de usuarios, en respuesta a sus dudas y necesidades informacionales, ya sea en el desarrollo de actividades sociales o profesionales así como en los trabajos rutinarios o en la generación de nuevas ideas y productos. La importancia de la información para el proceso de desarrollo fue objeto de consideraciones por parte del Rector de la Universidad Estatal de Campinas, Carlos Vogt, en el periódico O Estado de São Paulo, el 9 de Septiembre de 1992. Según él, los países que hicieron la revolución tecnológica de los últimos 15 años saben perfectamente que en la planilla de costos de cualquier producto - $\mathrm{e}$ incluso de un producto esencialmente intelectual- el inciso más valioso es el de la información agregada para el desarrollo de ese producto o servicio. La distancia que separa a los países llamados en desarrollo de los países desarrollados, ${ }^{2}$ depende menos de una cuestión de capacidad material que del acceso y dominio intelectual sobre la información.

El concepto de modernidad echa sus raíces en la cuestión del acceso a la in-

\footnotetext{
* Consultora Independiente e Investigadora Señor de la Universidad de Brasilia, Brasil.
} 
formación, sea ésta científica, tecnológica o cultural. En ese aspecto, es significativo notar que los sistemas de registro y de recuperación de documentos se encuentran en Brasil, a una distancia media de dos décadas con relación al Primer Mundo, intervalo superior o equivalente a los que separa nuestra industria de los Parques Fabriles más actualizados (Vogt, 1992). El papel de los sistemas de registro, de recuperación de acervos y de diseminación de la información es considerable. El documento y la información, al ser materias primas abundantes y en crecimiento, traen para las unidades informacionales muchos problemas de orden técnico y financiero. El problema de mantenimiento, actualización y desarrollo de las colecciones tal vez sea el mayor de ellos, al tener el aspecto completamiento de las colecciones deja de ser objetivo individual de las bibliotecas. El mito de la biblioteca autosuficiente, por lo tanto se desmoronó hace mucho tiempo.

Cada año se publican en el mundo cerca de 700 mil nuevos títulos de libros y a estos se puede aumentar el número inconmensurable de otros ítems bibliográficos, como panfletos, periódicos, documentos impresos, informes técnicos y de investigación, cintas video, mapas, bases de datos, normas técnicas, manuales técnicos, patentes, etcétera.

Esos nuevos ítems incorporados a la producción bibliográfica existente, forman un océano de más de 70 millones de títulos dispersos por los continentes del mundo (Mosher, 1986, p.21).

Frente a los problemas cuantitativos referentes a la producción bibliográfica y a la escasez de recursos para la adquisición, se pueden hacer las siguientes preguntas a bibliotecarios y profesionales de la información:

- ¿Cómo llegar a la actualización y, en la medida de lo posible al completamiento de las colecciones con relación a la producción documental?
- ¿Cómo conocer la disponibilidad informacional existente en el país y en el exterior?

- ¿Cómo hacer el control bibliográfico de los ítems producidos a nivel local, nacional e internacional?

- ¿Cómo localizar y tener acceso a los ítems existentes siempre que sean solicitados por los usuarios?

La preocupación por el control, la localización y el acceso al documento a nivel económico y administrativo, llevó a la conclusión de la necesidad de la cooperación con miras a compartir y utilizar al máximo los recursos existentes. Tal preocupación aún es válida para la última década antes de que completemos el segundo milenio, dado que el proceso de producción bibliográfica y documental no paró. Cada vez más se producen documentos e informaciones, ya se a de manera tradicional, con base en datos o en CD-ROM.

Debido al volumen de información producida, solamente se encontrará solución a los problemas antes señalados con el empleo de las nuevas tecnologías.

Las bibliotecas, tradicionalmente consideradas intermediarias entre productores, editores y usuarios de la información, no actualizaron sus concepciones ni adoptaron, en su mayoría, en cantidad y calidad, suficientes y nuevas tecnologías disponibles para desempeñar su papel en la cadena de la información. Esto se aplica principalmente a las bibliotecas en los países en desarrollo. Muchas de ellas no pudieron arrastrar los cambios para una metodología más moderna que permitiera manejar con eficiencia y rapidez un mayor número de informaciones.

A pesar de que el documento y la información lato sensu hubieran tenido un carácter sin fronteras, especialmente en estos tiempos en que se habla de comunicación, cultura, economía e información global (Toffler, 1990), los usuarios, culturalmente cosmopolitas, requieren cada vez más acceso a los recursos informacionales internaciona- les, lo que demanda redes y sistemas automatizados y accesibles en línea.

Una cooperación interbibliotecaria de este porte es el único medio para permitir el acceso a la información en escala internacional, Para alcanzar esta finalidad, los sistemas exigen tecnologías de información y redes de telecomunicación modernas, así como mediadas de normalización de carácter técnico, legal y administrativo.

El tema "think globally, act locally", está siendo interpretado por los países como un esfuerzo de integración comunitaria, y esta integración está dividiendo el mundo en diversos centros unidos por intereses económicos, culturales, étnicos y otros.

La nueva configuración geopolítica, cuyo foco de atención es la unión de países europeos en el Mercado Común, incluye la integración regional de otros países, como los del continente americano que han intentado constituirse en grupos: Estados Unidos, Canadá y México están formando un grupo de países norteamericanos; el Grupo de los Países Andinos, iniciado entre Colombia y Ecuador, hoy expande el libre comercio a Bolivia también; y el grupo de los países del Cono Sur, el MERCOSUR, que está siendo formado, inicialmente por Brasil, Argentina, Uruguay y Paraguay. ${ }^{3}$

A pesar de que la regionalización de la información no haya sido mencionada en términos oficiales, cabe en este contexto de cambios, a esos sistemas, asumir el papel de actores en la nueva sociedad de la información global y la iniciativa para globalizar e internacionalizar recursos y servicios. ¿Por dónde debemos empezar?

Un ejemplo que nos adviene del papel de la biblioteca/unidad de información, en el "nuevo orden mundial", es el de la Comunidad Europea, que para 1993 proyecta la unificación de acervos, productos y servicios.

La Comisión de las Comunidades Europeas (CCE) reconoció, hace tiempo que para que las bibliotecas cumplan su función a nivel comunitario, se necesita una acción basada en la cooperación in- 
ternacional y centrada en la aplicación de las más modernas tecnologías de la información y comunicación.

En 1985, una Resolución del Consejo de Ministros de la Comunidad, que reconocía la importancia de las bibliotecas como fuerza intrínseca en el mercado de la información intermediaria de la cultura y del conocimiento, invitó a la Comisión para actuar en el sendito de ayudar a las bibliotecas. Se elaboró un programa de trabajo para llevar a término algunas acciones preparatorias y exploratorias tales como proyectos piloto, estudios sobre últimos adelantos, etcétera. En total se registraron más de 60 acciones (10 acontecimientos, 10 proyectos preparatorios y 40 proyectos individuales).

Mientras tanto, para el lanzamiento definitivo del Programa de bibliotecas se requería una decisión del Consejo, que el 7 de junio de 1991 adoptó el programa comunitario específico de investigación y desarrollo tecnológico para el sector de sistemas temáticos en general. Dentro del programa se destinó un presupuesto de 22.5 millones de ecos (la moneda de la comunidad euro$\mathrm{pea}^{4}$ ) para las bibliotecas que constituyen una de las siete áreas cubiertas por el programa (junto con telemática administrativa, transporte, salud, educación a distancia, zonas rurales, investigación lingüística e ingeniería). Después que el Consejo adoptó el programa, se publicó en julio de 1991 la primera convocatoria para proyectos cooperativos, señalando como fecha límite de entrega el 2 de diciembre.

Con los principales objetivos del programa se pretende fomentar:

- La disponibilidad y accesibilidad de los servicios bibliotecarios modernos en toda la Comunidad Europea.

- La difusión más rápida y rentable de las nuevas tecnologías de información bibliotecaria.

- La normalización requerida para poder compartir los recursos;

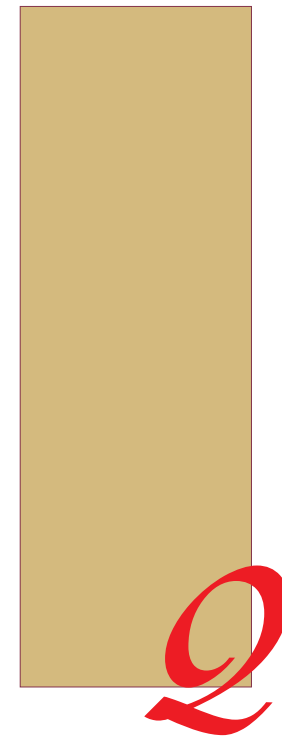

- La armonización convergencia de las políticas nacionales.

Estructuralmente, el programa comprende cuatro líneas de acción:

- Bibliografías informatizadas, el compartir los recursos bibliográficos puede ser comprometido, sí los datos bibliográficos en el intercambio no pudieran ser leídos automáticamente.

- Conexión internacional de sistemas y de normas internacionales a pesar de ser su objetivo final, se pretende a título experimental, apoyar proyectos -piloto, abarcando dos o más sistemas de bibliotecas con fines específicos, al utilizar alternadamente, redes de investigación de valor agregado (VANs) u otras redes de datos públicos. Se necesitará el apoyo de la industria, incluyendo el desarrollo de "hardware" y "software".

- Provisión de nuevos servicios bibliotecarios que utilicen tecnología de la información y comunicación. Esta línea de acción está dirigida a las pequeñas bibliotecas debido a su dificultad para justificar en términos financieros, nuevos servicios para los cuales la demanda es incierta. Se pretende crear servicios innovadores y experimentales, basados en nuevas tecnologías y podrán demostrar los beneficios derivados de dichos servicios.

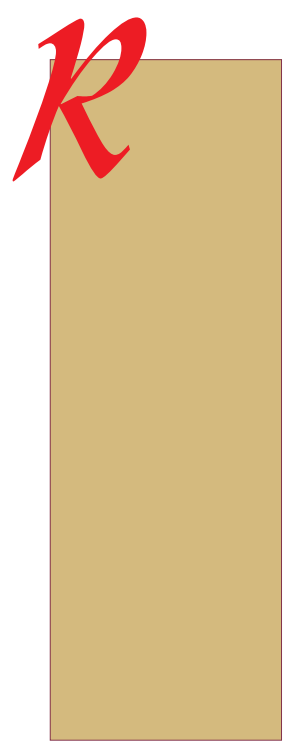

- Estimulación del mercado europeo para productos telemáticos y servicios específicos para bibliotecas —aquí reside la oportunidad de la Comunidad Europea para empezar a reorganizar su posición actual en términos de proveedor de productos y de servicios comercialmente viables. Actualmente cerca del 50\% de los sistemas automatizados de las bibliotecas son provistos por organizaciones ajenas a la Comunidad.

La acción comunitaria, dentro de los programas bibliotecarios, puede ser considerada como estímulo o catalizador de las actividades necesarias para provocar muchos de los cambios nece- 
sarios que vengan a concretar la internacionalización de la información, de su control y acceso. El resto dependerá de las bibliotecas de los Estados miembros. ¿Aprovecharán la oportunidad de modernizarse para hacer frente al año 1993 y sus consecuencias? (Ilion, 1991).

La propuesta del MERCOSUR aún no contempla la cuestión de la información o el problema de las bibliotecas como actores en el registro, recuperación y disminución de la información, factor vital con el adviento de la Sociedad de la Información. ${ }^{5}$ Queda, mientras tanto, el ejemplo y la experiencia de quien está al frente de ese proceso.

Las iniciativas de internacionalización, involucrando a pueblos de culturas, sociedades y economías diferentes, necesitan de la intervención y estimulo de los gobiernos y de las instituciones para normalizar, legalizar y administrar los cambios, así como del involucramiento de las comunidades regionales que les atañe. Las medidas de implementación deben descansar en el conocimiento tecnológico propio integrado y en la utilización de los medios electrónicos y de comunicación. No se debe privar de asistencia a las bibliotecas que no pudieran integrarse y participar directamente en el sistema.

Dentro de la perspectiva de internacionalizar la información en ocasión de la unificación del MERCOSUR, el papel de las unidades de información nos parece obvio, el de ser actores en el proceso de unificación dadas las disponibilidades y limitaciones locales. Es necesario verificar los problemas y potencialidades locales y regionales. En principio, los componentes del eje de la cooperación internacional son los mismos entre los países de los llamados Primer Mundo y del Tercer Mundo: acervos, utilización de nuevas tecnologías y el trabajo cooperativo (integrando adquisición, control bibliográfico y acceso al documento). No obstante, en primer lugar se deben resaltar las diferencias entre la dimensión de los acervos disponibles en los países del Primer Mundo, y aquella de los países del Tercer Mundo. Allá, prácticamente se adquiere todo lo que se edita, mientras que aquí se trabaja con exigüidad de recursos y toda suerte de limitaciones. En segundo lugar, la cuestión de las tecnologías de acceso, aún incipientes entre nosotros y allá ya alcanzan proporciones de industria próspera y rentable. Por último, tenemos el concepto, ya altamente difundido en el mundo industrializado de trabajo cooperativo y uso integrado de acervos, realidad que permea actualmente la universidad, la industria, los servicios liberales y los medios de comunicación ( $c f$. Vogt, 1992).

Con este trabajo se pretende destacar algunas iniciativas y tendencias que visan al desarrollo de los servicios informacionales en América Latina. Se tratará en especial del programa de acceso al documento primario COMUTEX una propuesta de programa cooperativo que podrá beneficiar, a través de la integración de informaciones y servicios, no sólo a los países integrantes del MERCOSUR, sino a los de toda América Latina, pudiendo llegar a representar un importante eslabón en la integración de la información a escala mundial.

\section{El COMUTEX}

Desde que el COMUT6 fue creado en Brasil, por el Decreto No. 456 del 5 de agosto de 1980, de la Secretaría de Educación y Cultura, como programa de acceso al documento, el COMUT se preocupó por el intercambio y acceso a documentos a nivel regional.

Algunas acciones fueron tomadas en este sentido, integrando varios países de la región en encuentros, cursos, seminarios y conferencias. Una de éstas, la $2^{\mathrm{a}}$. Conferencia Iberoamericana sobre Información y Documentación Científica y Tecnológica, REUNIBER II, realizada en Buenos Aires del 16 al 20 de noviembre de 1981, organizada por el Centro Argentino de Información Científica y Tecnológica (CAICYT) del Consejo Nacional de Investigación Científica y Técnica (CONICIT), con la colaboración del Instituto de Información y Documentación en Ciencia y Tecnología (ICYT) de España y asistencia de la Unesco. Participaron representantes de insti- tuciones iberoamericanas y de organismos regionales e internacionales que desarrollan actividades de información científica y tecnológica.

La REUNIBER II fue convocada para evaluar los resultados de los trabajos realizados como complemento a la 1a. Conferencia Iberoamericana, que se realizó en Madrid en 1978, con el fin de acorar futuras actividades cooperativas, para lo que se fijaron los siguientes temas prioritarios.

- Utilización de redes o sistemas de información por teleprocesamiento;

- acceso al documento primario;

- encuesta, control e inventario de las fuentes de información primarias Iberoamericanas.

El trabajo referente al segundo tema fue encomendado al Instituto Brasileño de Información en Ciencia y Tecnología (IBICT) de Brasil. Este trabajo presentado por Antonio Miranda, fue debatido y después de la constatación de que en le curso de la próxima déca $\mathrm{da}^{7}$ existirán, en todos los países miembros, mecanismos necesarios para facilitar el acceso al documento. En un esfuerzo de cooperación regional, reduciendo a la dependencia actual de otros centros, se fijaron las siguientes acciones:

- desarrollar la compilación de repertorios de instituciones de la región que proveen diversos tipos de documentos primarios originales, sus copias o traducción. Estos repertorios deberían indicar los tipos de servicios, condiciones, etcétera.

- Promover la actualización permanente de los catálogos para facilitar su difusión entre los países de la región.

- Promover la utilización de los medios de transmisión actuales (por ejemplo, telex, telecopiadora telefónica, etcétera), para la obtención del documento primario, y seguir con atención los avances tecnológicos en materia de transmisión a distancia de tales documentos; estudiar 
a nivel nacional e internacional mecanismos de desburocratización de procesos administrativos, así como de las comunicaciones postales para agilizar el intercambio de documentos en la región;

- Buscar un mecanismo para facilitar el acceso a la literatura no convencional, ${ }^{8}$ producida por las instituciones de los países iberoamericanos.

Para dar continuidad a las acciones propuestas, el CAICT de la Argentina quedó encargado de formar un grupo de trabajo para estudiar y discutir el acceso al documento primario. Éste, creado durante la Asamblea General Regional de la FID/CLA en 1982, fue precedido por un Seminario sobre Conmutación Bibliográfica que permitió fijar con mayores detalles a sus funciones. Este grupo fue denominado FID/CLA/CB y su coordinación quedó a cargo de Brasil.

El Seminario sobre Conmutación Bibliográfica, realizado en Buenos Aires el 6 y 7 de diciembre de 1982, discutió varios asuntos entre ellos cuatro modelos estructurales de sistemas de conmutación bibliográfica:

- una colección centralizada, proveedora del sistema;

- dos o tres grandes bibliotecas como principales fuentes de provisión;

- colecciones distribuidas entre un número mayor de bibliotecas creadas con ese propósito;

- colecciones totalmente descentralizadas en muchas bibliotecas.

Se discutieron tres tipos de conmutación bibliográfica:

- centralizada;

- parcialmente descentralizada

- descentralizada
Los componentes de la infraestructura operacional de un sistema de Conmutación Bibliográfica fueron enlistados, a saber:

- catálogos colectivos;

- equipo reprográfico;

- formatos normalizados;

- canales de comunicación;

- manual de procedimientos;

- vínculo legal;

- centro coordinador;

- cupones;

- recursos humanos;

- colecciones.

El grupo de trabajo FID/CLA/CB debería asumir las funciones básicas referentes a la:

- realización de estudios de usuarios, de costo/beneficio, encuestas de infraestructuras para conocer las posibilidades de la conmutación en América Latina.

- Divulgación de estos estudios a través de revistas y otros vehículos dedicados a la divulgación de los asuntos relacionados con América Latina. Ésta fue lanzada en la Revista Latinoamericana de Documentación que tuvo continuidad en el periodo en que la presidencia de la FID/CLA estuvo en Brasil (1981-1983);

- Elaboración de pautas y directrices;

- Formación de recursos humanos dirigidos a:

$\checkmark$ Los propios usuarios.

$\checkmark$ Autoridades.

$\checkmark$ Bibliotecarios profesionales.

$\checkmark$ Auxiliares técnicos.

- Promoción de reuniones para intercambiar experiencias.

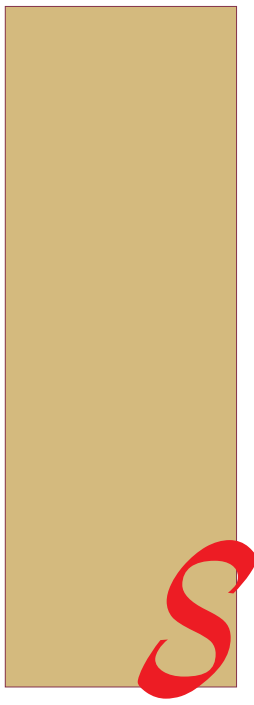

Dentro de la perspectiva de internacionalizar la información en ocasión de la unificación del MERCOSUR, el papel de las unidades de información nos parece obvio, el de ser actores en el proceso de unificación dadas las disponibilidades $y$ limitaciones locales. 
- Promoción de acciones para la formación de un Sistema de Conmutación Bibliográfica para América Latina, con la sigla COMUTEX (Seminario, 1983).

En 1984, dos misiones de asistencia técnica fueron enviadas a Colombia para el sistema de Información y Documentación para la Educación Superior (SIDES), del Instituto Colombiano para el Fomento de la Educación Superior (ICFES) y a Chile (Sistema universitario) para el desarrollo de la infraestructura del COMUT. Durante el mismo año se impartió en el Consejo Nacional de Información Científica y Tecnológica (CONICIT) de Venezuela, un curso de acceso a los documentos. En 1987 hubo un encuentro en Buenos Aires sobre el asunto, promovido por la Reunión Nacional de Bibliotecas Universitarias (RENBU) y por el IBICIT, con la participación de especialistas de Brasil, Argentina y Colombia (National, diciembre 1991).

El evento más reciente tuvo lugar del 13 al 17 de mayo de 1991, cuando se convocó en Caracas la Reunión de Consulta promovida por la INFOLAC-FID/CLA. El objetivo central de esta reunión fue analizar la necesidad de un programa de conmutación bibliográfica para la región -América Latina y Caribe-, a partir de análisis de las experiencias de los países integrantes. Se analizaron los siguientes puntos:

- ¿por qué realizar la conmutación?;

- ¿para qué un sistema de esta naturaleza?;

- Condiciones para establecer acciones de cooperación bilateral;

- Estructura de base con la cual se contaba;

- Capacidad para diseminar las publicaciones de cada país;

Participaron en la reunión: Argentina, Brasil, Colombia, Costa Rica, Cuba, Chile, Ecuador, Jamaica, Uruguay y Venezuela. Cada país hizo un relato de experiencia y se concluyó que los pro- blemas más comunes eran los de infraestructura:

- demoras en el envío por correo;

- costos de envío;

- costos asociados con el servicio;

Los problemas señalados en esa reunión son, básicamente, idénticos a los de la red brasileña y demuestran apenas que los sistemas aun no están aptos para una cooperación regional. A esto se aúna los catálogos colectivos incompletos y su difícil mantenimiento. La cuestión del catálogo colectivo es de fundamental importancia para la verbalización de la propuesta COMUTEX, deberá antes de otra cosa, garantizar el acceso a copias de documentos con buen servicio y en el menor tiempo posible. Asimismo, debería simplificar los pagos y procesos, racionalizar las adquisiciones, permitir la provisión de servicios más económicos y rápidos. Dichas conclusiones, la mayoría de ellas de carácter operacional, destacan la necesidad de la racionalización de adquisiciones, lo cual introduce una ponderación de carácter estratégico-táctico. La cooperación, sea ésta a nivel local o regional, debe ser también una cooperación técnica, integrando otros elementos del ciclo documental. En el centro de la cuestión de los acervos y la cuestión que se presenta es: si hubiera cooperación en la cuestión del acceso al documento, porqué no adoptar una adquisición cooperativa, ${ }^{9}$ para un desarrollo más integrado y armónico de los acervos, buscando mayor complementación y alcance. La otra vertiente del problema es la cuestión del control bibliográfico, cuyo instrumento principal a nivel nacional y regional es el Catálogo Colectivo.

Esa reunión destacó que el requisito básico para participar en el proyecto COMUTEX es el Catálogo Colectivo de Publicaciones periódicas, a nivel de una biblioteca, de un grupo de bibliotecas seleccionadas o de bibliotecas que representaran un sector especializado de la información, como el de las áreas de la salud, coordinado en Brasil por la Biblioteca Regional de Medicina (BIREME), que ya está respondiendo a solicitudes de copias de un buen número de países de América Latina.

Se sugirió un periodo experimental de dos años, señalando diciembre de 1991 como la fecha para el primer intercambio de los Catálogos Colectivos en soporte magnético, microfichas o papel entre los países que en esa reunión demostraron interés en el acuerdo de conmutación bibliográfica regional: Brasil, Costa Rica, Chile, Colombia, Cuba, Argentina y Venezuela. Esta acción aún no fue concretizada, sin embargo, está en marcha el proyecto CAPSALC Catálogo Colectivo de Publicaciones Seriadas de América Latina y del Caribe, que está desarrollándose en el Instituto Brasileño de Información en Ciencia y Tecnología IBICT, que pretende ofrecer un mecanismo eficiente para la conmutación bibliográfica entre las instituciones de la región.

\section{CAPSALC}

La idea de formar un catálogo colectivo de publicaciones seriadas para la región surgió en 1960, durante la 26a Reunión General de la FID, en Río de Janeiro. Esta acción también fue recomendada por la Unesco, en el Seminario Latinoamericano de Bibliografía, Documentación y Canje de Publicaciones, realizado en México en noviembre del mismo año.

El Instituto Brasileño de Bibliografía y Documentación (BBD, actual IBICT) quedó encargado de recibir las informaciones de las bibliotecas que quisieran cooperar y ejecutar las tareas de organización y divulgación del catálogo denominado Catálogo Colectivo de Publicaciones Periódicas de América Latina, CAPPAL. Participaron bibliotecas de Argentina, Chile, Colombia, Guatemala, México, El Salvador y Venezuela. En esta primera etapa se optó por no exigir uniformidad para el registro de los datos en el catálogo.

En 1962, el IBBD, bajo los auspicios de la FID/CLA, publicó una edición del CAPPAL. Esta publicación debería ha- 


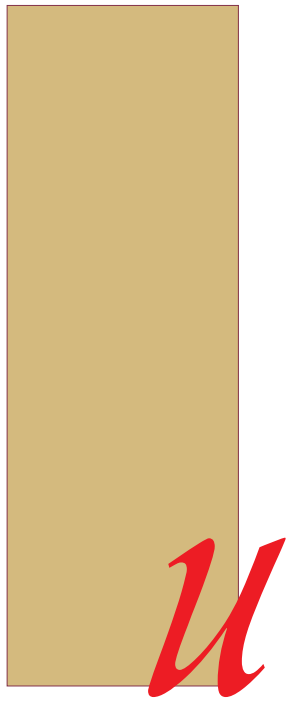

Los problemas más comunes para la conmutación bibliográfica, según se desprende de las experiencias dadas a conocer por diez. países latinoamericanos en la Reunión de consulta, son los relacionados con la infraestructura

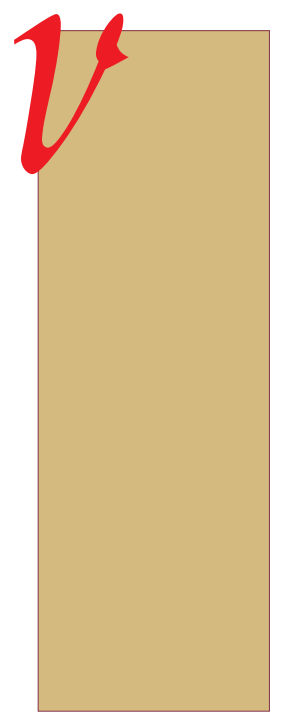

ber sido actualizada cada año, sin embargo, lamentablemente no tuvo continuidad.

El asunto fue retomado en 1987, en la reunión del Catálogo Colectivo Regional de Publicaciones Seriadas, realizada en Brasilia bajo los auspicios de la Organización de los Estados Americanos (OEA) y la Unesco. Esa reunión coordinada por el IBICT, contó con los representantes de los Catálogos Nacionales de Argentina, Brasil, Colombia, Chile, México, Uruguay y Venezuela.

También estuvo presente un representante del Centro Internacional del ISDS International Serials Data System (Sistema Internacional de Datos de Series). El Instituto Brasileño de Información en Ciencia y Tecnología (IBICT), utiliza en su Sistema Integrado de Publicaciones Seriadas (SIPS) para la entrada de los datos en el Catálogo Colectivo Nacional de Publicaciones Seriadas, un formato compatible con los formatos ISDS y BIBLIODATACALCO y, consecuentemente con el formato MARC II (Robredo y Cunha, 1986, p. 168).10

Las recomendaciones de esa reunión enfatizaron la adopción de patrones comunes para el intercambio de datos del catálogo Colectivo de publicaciones Periódicas para América Latina y el Caribe, CACALC, integrando parte de los catálogos de Argentina, Brasil, Colombia y México. Hecha una selección previa de 5 mil títulos de periódicos, la construcción de este núcleo obedecería a las siguientes etapas:

- Los países enviarían al IBICT los datos. Para el intercambio de datos se utilizaría el formato de títulos acordados en la reunión (SDS, modificado).

- El IBICT debería hacer la consolidación de estos datos y enviar a cada país la base de datos de títulos, completa.

- Para los títulos existentes en la base, los países enviarían al IBICT la descripción de los acervos de diez bibliotecas que estuvieran en condi- ciones de realizar la conmutación bibliográfica a nivel regional. Se utilizarían las normas de transcripción de datos para intercambio del IBICT;

- El IBICT integraría estos datos y enviaría a los países cooperantes la base de datos completa de los acervos.

Para facilitar el desarrollo de ese proyecto piloto, se decidió trabajar solamente con títulos que tuvieran ISSN (SCT/PR/CNPQ/IBICT, 1989).

El proyecto piloto no se desarrolló de la manera esperada. Entre los problemas encontrados para el cumplimiento de las etapas antes descritas se citan los siguientes:

- Los países no enviarían los datos de acuerdo con los formatos estipulados, Así, para cumplir con el objetivo propuesto, el IBICT tuvo que desarrollar programas específicos para identificar los datos de cada archivo, lo cual requirió una cantidad de trabajo apreciable, para etapa del proyecto piloto, que originalmente no había sido previsto;

- Los datos enviados no contenían los campos mínimos definidos como obligatorios en la Reunión.

- Los recursos de la OEA no pudieron utilizarse para la contratación de personal local, para apoyar los trabajos desarrollados por el IBICT, por ciertas restricciones de nivel interno.

A pesar de los percances el IBICT está con el formato CAPSALC, prácticamente desarrollado, habiendo utilizado en su prueba piloto el núcleo básico de los 5 mil títulos seleccionados, Para esto, se utilizó par ala colección el formato N/ISO Z3944; para la biblioteca del formato SIIR (formato de intercambio de informaciones, un referencial desarrollado en América Latina bajo la tutela de la Unesco); para el título del periódico el ISDS. 
La existencia de esa metodología posibilitará la elaboración del catálogo colectivo regional, el CAPSALC.

Basado en este catálogo, el INICT, apoyado por la UNESCO, intenta desarrollar otros proyectos piloto para la región, integrando por lo menos tres países para iniciar efectivamente el intercambio de fotocopias de documentos (National, 1991).

\section{CONCLUSIONES}

Existe interés por acciones cooperativas en la región. Las propuestas de mayor alcance para América Latina y el Caribe, nos parecen aún a nivel de discurso, aunque se estén tomando algunas acciones, como el CAPSALC y el COMUTEX. Los problemas que necesitan solución, no sólo para viabilizar la cooperación regional, sino para agilizar la provisión de servicios en los propios países participantes, se refieren a dificultades de organización y funcionamiento. Las dificultades de organización y funcionamiento. Las dificultades financieras comunes en la región, muchas veces han impedido el mantenimiento y actualización de los catálogos colectivos, instrumentos básicos para iniciar un programa de intercambio de documentos, habiendo provocado atrasos e interrupciones en la provisión de los servicios, a nivel local.

Para pensar en un intercambio/cooperación a nivel regional, parecen más factibles las acciones bilaterales, bajo la forma de proyectos-pilotos o programas un poco más ambiciosos, dentro del espíritu del MERCOSUR, integrando a Argentina, Uruguay, Paraguay y Brasil, donde cada uno asumiera la responsabilidad de organizar y permitir el acceso a su catálogo colectivo, en el menor plazo posible.

Tomando como ejemplo la experiencia del Mercado Común Europeo, se podrían tomar otras acciones a partir de este primer acuerdo comunitario, entre éstas:
- normalización de la documentación, adopción de entradas únicas para le material bibliográfico;

- utilización del mismo lenguaje de búsqueda y recuperación para la legibilidad de las referencias;

- implantación de una legislación de cooperación vía telecomunicaciones;

- mejor conocimiento de las disponibilidades de acervos, por áreas, en los diversos países participantes;

- encuesta, diagnóstico y solución de los problemas de infraestructura, operacionales y de funcionamiento;

- actualización inmediata y acceso a todos los catálogos colectivos, de preferencia, vía Terminal de conmutador y/o en CD-ROM;

- estudio de medios automatizados, económicamente accesibles y rápidos para el acceso a los documentos, como el FAX, el BITNET, y otros;

- estudio de la posibilidad de instalar un centro coordinador para las iniciativas de cooperación a nivel nacional, intentando viabilizar también la adquisición planificada;

- estudiar la posibilidad de instalar una sede común de donde puedan emitirse directrices y políticas así como la coordinación de los programas cooperativos regionales;

- adopción de una política financiera y moneda referencial única para el intercambio, y en el caso del COMUTEX, de cupones con valor referencial y efectivo único, basado en el indicador más estable, como por ejemplo, el dólar.

Solucionados algunos problemas básicos y otros específicos del CAPSALC y del COMUTEX, tal vez la realización del sueño de la cooperación comunitaria y de la regionalización de la información no esté tan lejos.

\section{LISTA DE SIGLAS}

BIBLIODATA-CALCO- es un sistema para la catalogación cooperativa en Brasil, desarrollado por la Biblioteca Central y el Centro de Procesamiento de Datos de la Fundación Getúlio Vargas.

BITNET- la sigla quiere decir literalmente: "Because it is timer to network". Su definición queda más clara a través de sus atribuciones: envío y recepción de cartas (el correo electrónico).

BN- Biblioteca Nacional.

CD-ROM-Compact Disc, Read Only Memory.

CAICYT- Centro Argentino de Información Científica y Tecnológica.

CACALC- Catálogo Colectivo de Publicaciones Periódicas para América Latina y el Caribe, sigla posteriormente substituida por la sigla CAPSALC;

CALCO- Catalogación Legible por Computadora. ES un formato basado en el MARC II de la Biblioteca del Congreso de los Estados Unidos desarrollado en Brasil.

CAPPAL- Catálogo Colectivo de Publicaciones Periódicas de América Latina, sigla posteriormente substituida por la sigla CAPSALC.

CAPSALC-CATÁLOGO Colectivo de Publicaciones Periódicas de América Latina y el Caribe.

CCE- Comisión de las Comunidades Europeas.

CEE-COMUNIDAD Europea formada por 12 naciones miembros: Alemania, Francia, Gran Bretaña, Dinamarca, Bélgica, Irlanda, Luxemburgo, Grecia, España, Italia, Holanda Portugal.

CIMEC- Centro de Informática de la Secretaria de Educación y Cultura, actualmente Secretaría de Informática, SEINF.

COMUT- Programa de Conmutación Bibliográfica para Brasil. La sigla sirve para designar el acto de una unidad de información al obtener material de otra unidad de información. 
COMUTEX- Conmutación Bibliográfica para América Latina.

CONACYT- Consejo Nacional de Ciencia y Tecnología (Ecuador)

CONICIT- Consejo Nacional de Investigación Científica y Técnica de Argentina; Venezuela adopta la misma sigla para designar el Consejo Nacional De Información Científica y Tecnológica.

FAX- es un tipo de equipamiento electrónico que puede mandar y recibir documentación a través de líneas telefónicas.

FGV- Fundación Getúlio Vargas.

FID- Federación Internacional de Documentación.

FID/CLA- Comisión Internacional de Documentación; FID/CLA/CB-GRUPO creado para estudiar y discutir el acceso al documento primario bajo la coordinación de la

FID/CLA; IBBD- Instituto Brasileño de Bibliografía y Documentación, Actual IBICT.

IBICT- Instituto Brasileño de Información en Ciencia y Tecnología.
ICFES- Instituto Colombiano para le Fomento de la Ecuación Superior.

ICYT- Instituto de Información y Documentación en Ciencia y Tecnología de España.

INFOLAC- Programa Regional para el Fortalecimiento de la Cooperación entre Redes y Sistemas Nacionales de Información para América Latina y el Caribe.

ISDS- International Serials Data System.

ISO- International Standard Organization.

ISSN- International Standard Serial Number.

MARC- Machine Readable Cataloguing. Formato desarrollado por la Library of Congress de los Estados Unidos.

MERCosur- Mercado Compón de Integración de los países del Cono Sur, inicialmente: Brasil, Argentina, Uruguay y Paraguay.

OEA- Organización de Estados Americanos.
RENBU- Red Nacional de Bibliotecas Universitarias de Argentina.

REUNIBER- Conferencia Iberoamericana sobre Información y Documentación Científica y Tecnológica.

SEINF: Secretaria de Informática.

SIDES- Sistema de Información y Documentación para la Educación Superior de Colombia.

SHR- Formato de intercambio de informaciones, referencial desarrollado en América Latina bajo la tutela de la Unesco.

SIPS- Sistema Integrado de Publicaciones Seriadas.

UNESCO- United Nations Education, Science and Culture Organization (Organización de las Naciones Unidas para la Educación, Ciencia y Cultura).

VANS- Redes de investigación de valor agregado.

\section{REFERENCIAS BIBLIOGÁFICAS}

DRUCKER. Peter. La sociedad del conocimiento. In: Drucker, Peter. Una era de doscontinuidad: orientaciones para una sociedad en cambio. Río de Janeiro: Ahar, 1976. p. 295-424.

FIGOLI, Silvia R. "Estudio comparativo de los informes presentados por los países iberoamericanos en REUNIBER II con relación al acceso al documento primario". Revista Latinoamericana de Documentación, v.3, n.2, p. 10-18, jul/dic. 1983.

FURTADO, Celso. Le mythe du developpement et le future du Tiers Monde, v. 15, p. 15-68, 1974.

ILJON, Ariane. "Estantería y Megabytes". XIII Magazine, Luxemburgo, n.4, p. 89, diciembre 1991. 
MOSHER, Paul H. "National Scheme for Collaboration in Collection Development: the RLG-NCIP Effort”. Resource Sharing and Information Networks, v.2, n 3/4, spring/summer 1985. C. 1986, National Information Network and Regional Interconnection. Brasilia: IBICT, December 1991 (Project Document).

NOCETTI, Milton. "La conmutación Bibliográfica". In: Machado, Waldino Dantas. Estudios avanzados en biblioteconomía y ciencia de la información. Brasilia: Asociación de los bibliotecarios del Distrito Federal, 1982. Cap. 6, p. 133-147.

PROGRAMA DE CONMUTACIÓN BIBLIOGRÁFICA COMUT. Proyecto. Brasilia: MEC/CAPES/CNPq/IBICT, diciembre de 1980.

REUNIÓN DE CONSULTA INFOLAC-FID/CLA. Caracas, 13-17 de mayo de 1991. Seminario de Conmutación Bibliográfica. Caracas, Mayo, 1991.

ROBREDO, Jaime y Cunha, Murilo B. da Documentación de hoy y de mañana. “ed. rev. y Ampl.. Brasilia: Edición del Autor, 1986.

SECRETARÍA DE CIENCIA Y TECNOLOGÍA, SCT/PR. Consejo Nacional de Desarrollo Científico y Tecnológico (CNPq). Instituto Brasileño de Información en Ciencia y Tecnología (IBICT). América Latina y Caribe un continente integrado en el uso de publicaciones periódicas. 3a. Reunión del CACALC, Brasilia, noviembre 1989 (uso cooperativo de publicaciones periódicas en América Latina y el Caribe).

SEMINARIO SOBRE CONMUTACIÓN BIBLIOGRÁFICA; Informa Resumido, Rev. LAt. Doc., v.3, n.1, p. 2-3, ene/jun. 1983.

TOFFLER, Alvin. Los cambios del poder; un perfil de la sociedad del siglo XXI por el análisis de las transformaciones en la naturaleza del poder. Trad. de Luiz Carlos do Nascimento Silva, revisión Técnica de Marcus Da Costa Moraes.

VOGT, Carlos. Universidad e Información O Estado de São Paulo, 9 de Septiembre de 1992, p.2.

Weitzen, H.S. El poder de la Información: cómo transformar la información que usted domina en un negocio lucrativo. São Paulo: Makron; McGraw-Hill, 1991. 


\section{NOTAS BIBLIOGRÁFICAS}

1. Valor es un concepto fundamental en Economía Política para designar el atributo que da a los bienes económicos. En Finanzas Públicas, el valor agregado es el total obtenido en la suma de las cuentas que representan determinado sector (Sandroni, Paulo. Diccionario de Economía, $5^{a}$ ed. revisado y ampliado, São Paulo: Best Seller, 1989. P. $321)$.

2. El autor usa la expresión "países centrales" de acuerdo con la teoría de desarrollo que distingue los países centrales dominantes, de los países periféricos dominados ( $c f$. Furtado, 1974, 60).

3. El MERCOSUR, pretende en cinco años, obtener una reducción gradual de tarifas y la libre comercialización de bienes y servicios, sin ninguna barrera aduanal.

4. El monto de 22.5 ecus corresponde aproximadamente a US $\$ 37.5 \mathrm{mi}$ llones. La adopción de la moneda única en substitución de las 12 unidades monetarias que existen actualmente en el ámbito de la CEE es el dispositivo más importante y complejo del Tratado de Maastritch sobre la unidad europea, negociado por los gobiernos de la organización de las 12 naciones miembros. La argumentación a favor de la moneda única es que una zona comercial, sin barreras internas para la movilización libre de bienes, personas y capital, no podría existir sin una moneda común aceptable por todos. Ese mercado deberá estar establecido el 1 de enero de 1993.

5. Sociedad de la Información es un concepto acuñado en la década de los 60, fue también designado como Sociedad del Conocimiento por Peter Drucker (1976), para caracterizar el desarrollo de un nuevo tipo de sociedad. Ésta tiene como sostén a la industria del conocimiento, es decir, la industria que produce y distribuye ideas e informaciones, teniendo como centro operativo ya no más la habilidad manual o mecánica, sino la capacidad de aplicar sus ideas e informaciones al sector productivo. Es la consecuencia del fortalecimiento del uso de los elementos que forman el triángulo de la información: computadora, teléfono e industria electrónica (Weitzen).

6. Esta sigla, así como el término conmutación bibliográfica, aparecen en el vocabulario biblioteconómico brasileño en la segunda mitad de la década de 70, sirviendo para designar el proceso de una unidad informacional para obtener material de otra unidad informacional por medio de reproducción fotográfica. La idea del aprovechamiento integral de las colecciones es subyacente a la de la filosofía de la conmutación (Nocetti, 1982, P. 134).

7. Para 1992, coincidiendo con el $5^{\circ}$ Centenario del Descubrimiento de América se propuso que todos los países de la región iberoamericana deberían alcanzar un nivel suficiente de desarrollo de sus infraestructuras de información para facilitar el acceso e intercambio de los documentos. 
8. Preferimos utilizar la expresión difundida en Brasil "literatura não convencional", mientras que en España y en América hispano-hablante parece prevalecer la expresión "literatura gris", equivalente a la "grey literature", utilizada en Inglaterra o aun a la "littèrature grise" en Francia, o "literatura cinzenta", utilizada en Portugal. Ya en Alemania existen ambas denominaciones, a saber: "grane literatur" y "nichtkonventionelle literatur".

9. El desarrollo de las colecciones hecho en colaboración es una experiencia practicada por la red de bibliotecas universitarias de Alemania, coordinada por el "Deutschesforchungsgemeinshaft", y también en el sistema de bibliotecas del Estado de Illinois, que a través del "Cooperative Collection Development Subcomittee" produjo en 1977.

10. "El formato CALCO está basado en el formato MARC II de la Biblioteca del Congreso de los Estados Unidos. Fue propuesto en 1972 por el IBBD, actualmente IBICI, y adoptado posteriormente por el Centro de Informática de La Secretaría de Educación y Cultura (CIMEC), actualmente Secretaría de Informática (SEINF), por la Biblioteca Nacional, y por la Fundación Getúlio Vargas (FGV). En 1977, la Biblioteca Nacional publicó in manual descriptivo intitulado Instrucciones de llenado de la hoja para catalogación CALCO, e inmediatamente otro manual denominado Instrucciones de llenado de hoja de entrada "CALCO autoridades", siguiendo de cerca la línea de la Biblioteca del Congreso de los Estados Unidos. A partir de esas dos publicaciones la Biblioteca Central y el Centro de Procesamiento de Datos de la fundación Getúlio Vargas iniciaron la implementación del Sistema Bibliodata-CALCO [...] El sistema se basa en la ISO 2709 de 1973, revisada en 1981, que es un formato para comunicación e intercambio de informaciones bibliográficas" (Robredo y Cunha, 1986, 9. 167, 110). 